ANNALES

POLONICI MATHEMATICI

$89.3(2006)$

\title{
Density of Morse functions on sets definable in o-minimal structures
}

\author{
by TA LÊ LOI (Dalat)
}

\begin{abstract}
We present a tameness property of sets definable in o-minimal structures by showing that Morse functions on a definable closed set form a dense and open subset in the space of definable $C^{p}$ functions endowed with the Whitney topology.
\end{abstract}

Introduction. In Morse theory it is proved that the topological shape of a space can be described via data given by Morse functions defined on the space. For Morse theory on compact smooth manifolds we refer the readers to the book by Milnor [Mi], and for Morse theory on singular spaces to the book by Goresky and MacPherson [GM]. [GM] proves the density and openness of Morse functions on closed Whitney stratified subanalytic sets in the space of smooth functions endowed with the Whitney topology (see also $[\mathrm{Mo}],[\mathrm{Mi}],[\mathrm{La}],[\mathrm{Be}],[\mathrm{P}],[\mathrm{O}]$ and $[\mathrm{Br}])$.

In this note we present similar results for definable sets in o-minimal structures. The definitions of o-minimal structures and Morse functions are given in Section 1, and the main theorems are stated and proved in Section 2. The proofs are based on Sard's theorem and an approximation theorem in the o-minimal context. The proofs of Proposition 1 and Theorem 2 are essentially the same as the corresponding proofs in [GM], with "subanalytic" replaced by "definable". Since the proofs are short, they are included for completeness. Note that the spiral $\left\{(x, y) \in \mathbb{R}^{2}: x=e^{-\varphi^{2}} \cos \varphi, y=e^{-\varphi^{2}} \sin \varphi\right.$, $\varphi \geq 0\} \cup\{(0,0)\}$ or the oscillation $\left\{(x, y) \in \mathbb{R}^{2}: y=x \sin (1 / x), x>0\right\} \cup\{(0,0)\}$ has no Morse functions, even though the first one is a closed Whitney stratified set (see Remark 1 in 1.4). Therefore, in some sense, our results show a tameness property of definable sets.

A part of this note was presented at the Conference on Real Algebraic Geometry and its Applications, at the ICTP, Trieste, 2003. The author

2000 Mathematics Subject Classification: 32B20, 14P10, 14B05.

Key words and phrases: o-minimal structures, Morse functions. 
thanks the institute and the organizers of the conference for invitation and hospitality.

1. Preliminaries. In this section, we recall some notions and notations, and give results needed to prove our main theorems.

1.1. O-minimal structures. The theory of o-minimal structures is a generalization of semialgebraic and subanalytic geometry. Here we only recall the definition. For the details we refer the readers to $[D],[D M]$ and $[C]$.

A structure on the real field $(\mathbb{R},+, \cdot)$ is a sequence $\mathcal{D}=\left(\mathcal{D}_{n}\right)_{n \in \mathbb{N}}$ such that the following conditions are satisfied for all $n \in \mathbb{N}$ :

- $\mathcal{D}_{n}$ is a Boolean algebra of subsets of $\mathbb{R}^{n}$.

- If $A \in \mathcal{D}_{n}$, then $A \times \mathbb{R}, \mathbb{R} \times A \in \mathcal{D}_{n+1}$.

- If $A \in \mathcal{D}_{n+1}$, then $\pi(A) \in \mathcal{D}_{n}$, where $\pi: \mathbb{R}^{n+1} \rightarrow \mathbb{R}^{n}$ is the projection on the first $n$ coordinates.

- $\mathcal{D}_{n}$ contains $\left\{x \in \mathbb{R}^{n}: P(x)=0\right\}$ for every polynomial $P \in \mathbb{R}\left[X_{1}, \ldots, X_{n}\right]$.

A structure $\mathcal{D}$ is said to be $o$-minimal if

- Each set in $\mathcal{D}_{1}$ is a finite union of intervals and points.

A set belonging to $\mathcal{D}$ is said to be definable (in that structure). Definable maps in $\mathcal{D}$ are maps whose graphs are definable sets in $\mathcal{D}$.

In recent years, many interesting o-minimal structures have been found, and it is shown that o-minimal structures share many important results with those of semialgebraic geometry or subanalytic geometry (see the survey $[\mathrm{DM}])$.

In this note we fix an o-minimal structure on $(\mathbb{R},+, \cdot)$. "Definable" means definable in this structure. Let $p$ be a positive integer, $p \geq 2$.

1.2. Definable stratifications. A definable $C^{p}$ Whitney stratification of $X \subset \mathbb{R}^{n}$ is a partition $\mathcal{S}$ of $X$ into finitely many subsets, called strata, such that:

- Each stratum is a $C^{p}$ submanifold of $\mathbb{R}^{n}$ and also a definable set.

- For every $S \in \mathcal{S}, \bar{S} \backslash S$ is a union of strata.

- For every $S, R \in \mathcal{S}$, if $S \subset \bar{R} \backslash R$, then $(S, R)$ satisfies Whitney's conditions $A$ and $B$ (defined in [Wh]).

We say that the stratification $\mathcal{S}$ is compatible with a class $\mathcal{A}$ of subsets of $\mathbb{R}^{n}$ if for each $S \in \mathcal{S}$ and $A \in \mathcal{A}, S \subset A$ or $S \cap A=\emptyset$.

The following theorem is proved in [Lo].

TheOREm 1. Given a finite collection $\mathcal{A}$ of definable sets in $\mathbb{R}^{n}$, there exists a definable $C^{p}$ Whitney stratification of $\mathbb{R}^{n}$ compatible with $\mathcal{A}$. 
1.3. Tangents to definable sets. Let $X$ be a definable subset of $\mathbb{R}^{n}$. Let $\mathcal{S}$ be a definable $C^{p}$ Whitney stratification of $X$. Note that if $S$ is a definable submanifold of $\mathbb{R}^{n}$, then the tangent bundle $T S$ and the cotangent bundle $T^{*} S$ are definable submanifolds of $T \mathbb{R}^{n}$ and $T^{*} \mathbb{R}^{n}$, respectively.

For $S \in \mathcal{S}$, the conormal bundle of $S$ in $\mathbb{R}^{n}$ is defined by

$$
T_{S}^{*} \mathbb{R}^{n}=\left\{(\xi, x) \in\left(\mathbb{R}^{n}\right)^{*} \times S:\left.\xi\right|_{T_{x} S}=0\right\}
$$

Note that $T_{S}^{*} \mathbb{R}^{n}$ is a definable submanifold of $T^{*} \mathbb{R}^{n}$ of dimension $n$.

A generalized tangent space $Q$ at $x \in S$ is any plane of the form

$$
Q=\lim _{y \rightarrow x} T_{y} R
$$

where $R \in \mathcal{S}$ and $S \subset \bar{R}$.

The cotangent vector $(\xi, x)$ is degenerate if there exists a generalized tangent space $Q$ at $x, Q \neq T_{x} S$, such that $\left.\xi\right|_{Q}=0$.

Proposition 1. The set of degenerate cotangent vectors which are conormal to $S$ is a conical definable set of dimension $\leq n-1$.

Proof. Let $R$ be a stratum in $\mathcal{S}$ with $S \subset \bar{R} \backslash R$, and $\operatorname{dim} R=r$. Consider the mapping

$$
g: R \rightarrow G_{r}\left(\mathbb{R}^{n}\right), \quad g(x)=T_{x} R
$$

(where $G_{r}\left(\mathbb{R}^{n}\right)$ denotes the Grassmannian of $r$-dimensional vector subspaces of $\mathbb{R}^{n}$ ). The graph $g$ of this mapping is a definable set of dimension $r$. So its closure $\bar{g}$ in $\mathbb{R}^{n} \times G_{r}\left(\mathbb{R}^{n}\right)$ is a definable set, and hence $\operatorname{dim}(\bar{g} \backslash g) \leq r-1$. Let

$$
A_{R}=\left\{(\xi, x, Q) \in T_{S}^{*} \mathbb{R}^{n} \times G_{r}\left(\mathbb{R}^{n}\right):(x, Q) \in \bar{g} \backslash g,\left.\xi\right|_{Q}=0\right\} .
$$

Then $A_{R}$ is definable. For each $(x, Q) \in \bar{g} \backslash g$ the fiber $A_{R} \cap\left(\mathbb{R}^{n}\right)^{*} \times(x, Q)$ has dimension $\leq n-r$. Hence, $\operatorname{dim} A_{R} \leq \operatorname{dim}(\bar{g} \backslash g)+(n-r)=n-1$.

Since there are a finite number of strata $R$ in $\mathcal{S}$ such that $S \subset \bar{R} \backslash R$, the set of degenerate cotangent vectors which are conormal to $S$ is of dimension $\leq n-1$.

1.4. Morse functions on stratified sets (cf. [Be], $[\mathrm{La}],[\mathrm{P}]$ and $[\mathrm{GM}]$ ). Let $X$ be a subset of $\mathbb{R}^{n}$ and $\mathcal{S}$ be a $C^{p}$ Whitney stratification of $X$.

A Morse function $f$ on $X$ is the restriction of a $C^{p}$ function $\tilde{f}: \mathbb{R}^{n} \rightarrow \mathbb{R}$ satisfying the following conditions:

(M1) For each $S \in \mathcal{S}$, the critical points of $\left.f\right|_{S}$ are nondegenerate, i.e. if $\operatorname{dim} S \geq 1$, then the Hessian of $\left.f\right|_{S}$ at each critical point is nonsingular.

(M2) For every critical point $x \in S$ of $\left.f\right|_{S}$, and for each generalized tangent space $Q$ at $x$ with $Q \neq T_{x} S,\left.d \tilde{f}(x)\right|_{Q} \neq 0$, i.e. $d \widetilde{f}(x)$ is not 
a degenerate cotangent vector (where $d \tilde{f}(x)$ denotes the derivative of $\widetilde{f}$ at $x$ ).

REMARK 1. The property of being a Morse function depends strictly on the particular stratification of $X$. For example, every zero-dimensional stratum is a critical point of $f$. We also note that all lines through $(0,0)$ are generalized tangent spaces of the spiral as well as of the oscillation mentioned at the beginning of this note. So there are no Morse functions on these sets.

REMARK 2. From the definition, one can check that if $\mathcal{S}^{\prime}$ is a definable $C^{p}$ Whitney stratification of $X$ compatible with $\mathcal{S}$, and $Z$ is a union of strata of $\mathcal{S}$, and $f$ is a Morse function on $\left(X, \mathcal{S}^{\prime}\right)$, then $f$ is Morse on $(X, \mathcal{S})$ and on $\left(Z,\left.\mathcal{S}\right|_{Z}\right)$.

2. Density of Morse functions on definable sets. Throughout this section, let $X$ be a definable closed subset of $\mathbb{R}^{n}$, which is endowed with a definable $C^{p}$ Whitney stratification $\mathcal{S}$. We will show that the set of Morse functions on $X$ is dense and open in the space of definable $C^{p}$ functions on $\mathbb{R}^{n}$, equipped with the definable Whitney topology (see 2.2 for the definition). The main results are Theorems 2 and 3 .

2.1. Parameter version of the density for Morse functions. Let $T$ be a definable $C^{p}$ manifold. Let $F: T \times \mathbb{R}^{n} \rightarrow \mathbb{R}, F(t, x)=f_{t}(x)$ be a definable $C^{p}$ function. Define $\Phi: T \times \mathbb{R}^{n} \rightarrow T^{*} \mathbb{R}^{n}$ by $\Phi(t, x)=\left(d f_{t}(x), x\right)$. Consider the set of "Morse parameters" $M(F, X)=\left\{t \in T:\left.f_{t}\right|_{X}\right.$ is a Morse function $\}$. Note that $M(F, X)$ is a definable set.

THEOREM 2. If $\Phi$ is a submersion, then $M(F, X)$ is an open subset of $T$ and $\operatorname{dim}(T \backslash M(F, X))<\operatorname{dim} T$.

Proof. For each $S \in \mathcal{S}$, set

$$
\begin{aligned}
& M_{1}=M_{1}(S)=\left\{t \in T:\left.f_{t}\right|_{S} \text { has nondegenerate critical points }\right\}, \\
& M_{2}=M_{2}(S)=\left\{t \in T: d f_{t}(x) \text { is a nondegenerate covector for each } x \in S\right\} .
\end{aligned}
$$

It is easy to check that $M_{1}$ and $M_{2}$ are definable sets.

Now we claim that $\operatorname{dim}\left(T \backslash M_{1} \cap M_{2}\right)<\operatorname{dim} T$. To prove this, let

$$
D=D(S)=\left\{(\xi, x) \in T_{S}^{*} \mathbb{R}^{n}: \xi \text { is a degenerate cotangent vector at } x\right\} \text {. }
$$

Then $D$ is a definable set. Let $\Phi_{S}: T \times S \rightarrow T^{*} S, \Phi_{S}(t, x)=\left(\left(d f_{t} \mid S\right)(x), x\right)$, and $\pi: T \times \mathbb{R}^{n} \rightarrow T$ be the natural projection. Since $\Phi$ is submersive, $\Phi_{S}$ is transverse to the zero section $S$ of $T^{*} S$. So the set $V_{1}=\Phi_{S}^{-1}(S)$ is a definable submanifold of $T \times S$. Furthermore, $t \in M_{1}$ if and only if $t$ is not a critical value of $\left.\pi\right|_{V_{1}}$. By Sard's theorem (see [Wi, Th. 2.7]), $\operatorname{dim}\left(T \backslash M_{1}\right)<\operatorname{dim} T$.

On the other hand, $\Phi$ is transverse to each stratum of any Whitney stratification of $D$, and by Proposition $1, \operatorname{dim} D \leq n-1$, so the set $V_{2}=$ 
$\Phi^{-1}(D)$ is a definable set of dimension $\leq \operatorname{dim} T-1$. So $\operatorname{dim}\left(T \backslash M_{2}\right)=$ $\operatorname{dim} \pi\left(V_{2}\right) \leq \operatorname{dim} T-1$.

Since the collection $\mathcal{S}$ is finite, the claim implies $\operatorname{dim}(T \backslash M(F, X))<$ $\operatorname{dim} T$. Openness of $M(F, X)$ follows from the second part of Theorem 3 below.

Corollary 1. Consider the square of distance function

$$
F: \mathbb{R}^{n} \times \mathbb{R}^{n} \rightarrow \mathbb{R}, \quad F(t, x)=\|t-x\|^{2} .
$$

Let $M=\left\{t \in \mathbb{R}^{n}: F(t, \cdot)\right.$ is a Morse function on $\left.X\right\}$. Then $M$ is definable, open and dense in $\mathbb{R}^{n}$.

Corollary 2. Let $f: \mathbb{R}^{n} \rightarrow \mathbb{R}$ be a definable $C^{p}$ function. Consider the linear deformations $f+L$, where $L$ is a linear form of $\mathbb{R}^{n}$. Let

$$
M=\left\{L \in L\left(\mathbb{R}^{n}, \mathbb{R}\right): f+L \text { is a Morse function on } X\right\} .
$$

Then $M$ is definable, open and dense in $L\left(\mathbb{R}^{n}, \mathbb{R}\right)$.

REMARK 3. Using the same arguments as in the proof of Theorem 2, we obtain the following proposition (cf. [S, Th. II.5.4(3)]).

Proposition 2. Let $M, N$ and $T$ be definable $C^{p}$ manifolds, and $\Phi$ : $T \times M \rightarrow N$ be a definable $C^{p}$ map. Let $\mathcal{A}$ be a finite collection of definable submanifolds of $N$, and $D$ be a definable subset of $N$ of codimension $\geq$ $\operatorname{dim} M+1$. If $\Phi$ is submersive, then the set

$$
\tau(\Phi, \mathcal{A}, D)=\{t \in T: \Phi(t, \cdot) \text { is transverse to } \mathcal{A} \text { and } \Phi(t, M) \cap D=\emptyset\}
$$

is a definable set and $\operatorname{dim}(T \backslash \tau(\Phi, \mathcal{A}, D))<\operatorname{dim} T$.

2.2. Density and openness of Morse functions in $\mathcal{D}^{p}\left(\mathbb{R}^{n}\right)$. Let $\mathcal{D}^{p}\left(\mathbb{R}^{n}\right)$ denote the space of definable $C^{p}$ functions on $\mathbb{R}^{n}$. On this space the definable Whitney topology is defined as follows.

Let $f \in \mathcal{D}^{p}\left(\mathbb{R}^{n}\right)$. For each positive continuous definable function $\varepsilon$ on $\mathbb{R}^{n}$, the $\varepsilon$-neighborhood of $f$ in this topology is defined by

$$
U_{\varepsilon}(f)=\left\{g \in \mathcal{D}^{p}\left(\mathbb{R}^{n}\right):\left|\partial^{\alpha}(g-f)\right|<\varepsilon, \forall \alpha \in \mathbb{N}^{n},|\alpha| \leq p\right\}
$$

where $\alpha=\left(\alpha_{1}, \ldots, \alpha_{n}\right) \in \mathbb{N}^{n},|\alpha|=\alpha_{1}+\cdots+\alpha_{n}, \partial^{\alpha} f=\partial^{|\alpha|} f / \partial x_{1}^{\alpha_{1}} \cdots \partial x_{n}^{\alpha_{n}}$.

Theorem 3. The set of definable $C^{p}$ functions on $\mathbb{R}^{n}$ which are Morse functions on $X$ and have distinct critical values is dense and open in $\mathcal{D}^{p}\left(\mathbb{R}^{n}\right)$.

Before proving the theorem, we prepare some lemmas.

Let $d(\cdot, \cdot)$ and $\|\cdot\|$ denote the distance and the norm on $\mathbb{R}^{n}$ induced by the Euclidean inner product, respectively. Let $L\left(\mathbb{R}^{n}, \mathbb{R}\right)$ denote the space of linear forms on $\mathbb{R}^{n}$, and $L_{2}\left(\mathbb{R}^{n}, \mathbb{R}\right)$ the space of bilinear forms on $\mathbb{R}^{n}$. For $L \in L\left(\mathbb{R}^{n}, \mathbb{R}\right), B \in L_{2}\left(\mathbb{R}^{n}, \mathbb{R}\right)$, and $T \in G_{k}\left(\mathbb{R}^{n}\right)$, as usual, we define

$$
\begin{aligned}
& \left\|\left.L\right|_{T}\right\|=\sup \{|L(v)|: v \in T,\|v\|=1\}, \\
& \left\|\left.B\right|_{T}\right\|=\sup \{|B(u, v)|: u, v \in T,\|u\|=\|v\|=1\},
\end{aligned}
$$


and det $\left.B\right|_{T}$ to be the determinant of the matrix representation of $\left.B\right|_{T}$ with respect to an orthonormal basis of $T$.

By the definition we have

LEMMA 1. The mappings

$$
\begin{aligned}
L\left(\mathbb{R}^{n}, \mathbb{R}\right) \times G_{k}\left(\mathbb{R}^{n}\right) & \rightarrow \mathbb{R}, \quad(L, T) \mapsto\left\|\left.L\right|_{T}\right\|, \\
L_{2}\left(\mathbb{R}^{n}, \mathbb{R}\right) \times G_{k}\left(\mathbb{R}^{n}\right) & \rightarrow \mathbb{R}, \quad(B, T) \mapsto\left\|\left.B\right|_{T}\right\|, \\
L_{2}\left(\mathbb{R}^{n}, \mathbb{R}\right) \times G_{k}\left(\mathbb{R}^{n}\right) & \rightarrow \mathbb{R},\left.\quad(B, T) \mapsto \operatorname{det} B\right|_{T},
\end{aligned}
$$

are continuous and semialgebraic.

Let $f \in \mathcal{D}^{p}\left(\mathbb{R}^{n}\right)$. To test the Morsity of $\left.f\right|_{S}$ at $x \in S \in \mathcal{S}$, we define

$$
m_{f, S}(x)=\left\|\left.d f(x)\right|_{T_{x} S}\right\|+\left|\operatorname{det} d^{2} f(x)\right|_{T_{x} S} \mid \frac{d(x, \partial S)}{1+d(x, \partial S)},
$$

where $d^{2} f(x)$ is the second derivative of $f$ at $x, \partial S=\bar{S} \backslash S$, and $d(x, \emptyset)=1$. Note that, by Lemma $1, m_{f, S}$ is a continuous definable function on $S$, and $\left.f\right|_{S}$ is Morse at $x$ if and only if $m_{f, S}(x)>0$. In general, $m_{f, S}$ cannot be continuously extended to the closure $\bar{S}$. Instead, for a Morse function $f, m_{f, S}$ is bounded from below by the restriction of a positive continuous function on $\mathbb{R}^{n}$, constructed as follows.

Lemma 2. Let $f \in \mathcal{D}^{p}\left(\mathbb{R}^{n}\right)$. Then $f$ is a Morse function on $X$ if and only if there exists a positive continuous definable function $m_{f}$ on $\mathbb{R}^{n}$ such that for each $S \in \mathcal{S}, m_{f}(x) \leq m_{f, S}(x)$ for all $x \in S$.

Proof. Assume $f$ is Morse on $X$. To construct $m_{f}$, we imitate the arguments of the proof of Lemma 6.12 in [C].

For each $S \in \mathcal{S}$, let

$$
\mu(r)=\inf \left\{m_{f, S}(x): x \in S,\|x\| \leq r\right\} .
$$

Then $\mu(r)$ is defined when $r \geq r_{0}$ for some $r_{0}>0$. So $\mu:\left[r_{0}, \infty\right) \rightarrow \mathbb{R}$ is a positive definable nonincreasing function. To prove $\mu(r)>0$, suppose to the contrary that $\mu(r)=0$. Then there exists a sequence $\left(x_{k}\right)$ in $S$ with $\left\|x_{k}\right\| \leq r$ and $m_{f, S}\left(x_{k}\right) \rightarrow 0$. By the boundedness, taking a subsequence if necessary, we can assume that $x_{k} \rightarrow x \in \bar{S}$ and $T_{x_{k}} S \rightarrow Q$. This implies

$$
\left\|\left.d f(x)\right|_{Q}\right\|+\left|\operatorname{det} d^{2} f(x)\right|_{Q} \mid \frac{d(x, \partial S)}{1+d(x, \partial S)}=0 .
$$

If $x \in S$, then $Q=T_{x} S$ and hence the above equality contradicts condition (M1).

If $x \in \partial S$, then $Q$ is a generalized tangent space. Since $X$ is closed and the strata of $\mathcal{S}$ satisfy the Whitney condition $A$, the above equality contradicts condition (M2).

By the monotonicity theorem (see [D, Th. 1.2, Ch. 3]), there exists $a \geq r_{0}$ such that $\mu$ is continuous on $[a, \infty)$. Let $\theta: \mathbb{R} \rightarrow[0,1]$ be a continuous 
nondecreasing definable function such that $\theta=0$ on $(-\infty, a], \theta=1$ on $[a+1, \infty)$. Define $\phi_{S}: \mathbb{R}^{n} \rightarrow \mathbb{R}$ by

$$
\phi_{S}(x)=\theta(\|x\|) \mu(\|x\|)+(1-\theta(\|x\|)) \mu(a+1) .
$$

Then $\phi_{S}$ is positive, continuous, definable and by construction $\phi_{S} \leq m_{f, S}$ on $S$. Define $m_{f}=\min \left\{\phi_{S}: S \in \mathcal{S}\right\}$. Then $m_{f}$ has the desired properties.

Now assume conversely that $m_{f}$ is a positive continuous definable function on $\mathbb{R}^{n}$ such that for each $S \in \mathcal{S}, m_{f}(x) \leq m_{f, S}(x)$ for all $x \in S$. Then $m_{f, S}(x)>0$, and hence $\left.f\right|_{S}$ satisfies condition (M1). On the other hand, if a sequence of points $x_{k} \in S$ converges to $y \in \partial S$, and $T_{x_{k}} S$ converges to $Q$, then the above inequality implies $\left\|\left.d f(y)\right|_{Q}\right\| \geq m_{f}(y)>0$, and hence $f$ satisfies condition (M2). Therefore, $f$ is Morse on $X$.

Roughly speaking, the following lemma says that in the $(\varepsilon, \delta)$-formulation of continuity, $\delta$ can be chosen to be continuously dependent on $\varepsilon$ and the variables.

Lemma 3. Let $\psi: F \rightarrow \mathbb{R}$ be a continuous definable function. Suppose $F$ is a closed subset of $\mathbb{R}^{n}$. Then there exists a positive continuous definable function $\delta: \mathbb{R}_{+} \times F \rightarrow \mathbb{R}$ satisfying

$$
x^{\prime} \in F,\left\|x^{\prime}-x\right\|<\delta(\varepsilon, x) \Rightarrow\left|\psi\left(x^{\prime}\right)-\psi(x)\right|<\varepsilon .
$$

Proof. Let

$$
\begin{aligned}
A=\{(\varepsilon, x, \delta): \varepsilon>0, & x \in F, \delta>0, \\
& \left.\left(\forall x^{\prime} \in F,\left\|x^{\prime}-x\right\|<\delta \Rightarrow\left|\psi\left(x^{\prime}\right)-\psi(x)\right|<\varepsilon\right)\right\} .
\end{aligned}
$$

Then $A$ is a definable set. For each $(\varepsilon, x) \in \mathbb{R}_{+} \times F$, define

$$
\bar{\delta}(\varepsilon, x)=\min \{\sup \{\delta:(\varepsilon, x, \delta) \in A\}, 1\} .
$$

Since $\psi$ is a continuous definable function, $\bar{\delta}$ is well defined, definable and positive on $\mathbb{R}_{+} \times F$. For $r>0$, define

$$
\mu(r)=\frac{1}{2} \inf \{\bar{\delta}(\varepsilon, x): \varepsilon \geq 1 / r, x \in F,\|x\| \leq r\} .
$$

Then $\mu:(0, \infty) \rightarrow \mathbb{R}$ is a definable nonincreasing function. Moreover, $\mu(r)>0$ for all $r>0$. Indeed, by the uniform continuity of $\psi$ on $\{x \in F$ : $\|x\| \leq r+1\}$, there exists $\delta_{0} \in(0,1)$ such that if $x, x^{\prime} \in F,\|x\| \leq r+1,\left\|x^{\prime}\right\| \leq$ $r+1$, and $\left\|x-x^{\prime}\right\|<\delta_{0}$, then $\left|\psi(x)-\psi\left(x^{\prime}\right)\right|<1 / r$. So, by the definition of $\bar{\delta}$, if $\varepsilon \geq 1 / r, x \in F$ and $\|x\| \leq r$, then $\bar{\delta}(\varepsilon, x) \geq \bar{\delta}(1 / r, x) \geq \delta_{0}>0$. Therefore, $\mu(r) \geq \delta_{0} / 2>0$.

Repeat the arguments of the proof of Lemma 2 for this $\mu$, keep the notations there, and then define $\delta: \mathbb{R}_{+} \times F \rightarrow \mathbb{R}$ by

$$
\begin{aligned}
\delta(\varepsilon, x)= & \theta(\max (1 / \varepsilon,\|x\|)) \mu(\max (1 / \varepsilon,\|x\|)) \\
& +(1-\theta(\max (1 / \varepsilon,\|x\|))) \mu(a+1) .
\end{aligned}
$$

It is easy to check that $\delta$ has the desired properties. 
LEMMA 4. Let $\varepsilon: \mathbb{R}^{n} \rightarrow \mathbb{R}$ be a positive continuous definable function. Then there exists a positive definable $C^{p}$ function $\varphi: \mathbb{R}^{n} \rightarrow \mathbb{R}$ such that

$$
\left|\partial^{\alpha} \varphi\right|<\varepsilon, \quad \forall|\alpha| \leq p
$$

Proof. For $n=1$, by the monotonicity theorem and approximation theorem (see [E, Th. 1.1]), we are reduced to proving the lemma for $\varepsilon$ being an even, strictly decreasing on $\mathbb{R}_{+}$, positive, $C^{p}$ definable function.

If $\lim _{t \rightarrow \infty} \varepsilon(t)>0$, then take $\varphi=c$, where $c$ is a constant, $0<c<$ $\min _{t \in \mathbb{R}} \varepsilon(t)$.

If $\lim _{t \rightarrow \infty} \varepsilon(t)=0$, then by the monotonicity theorem, $\varepsilon^{\prime}$ is strictly increasing on a neighborhood of $\infty$, and tends to 0 at $\infty$. Repeating the previous argument for $\varepsilon^{\prime \prime}, \ldots, \varepsilon^{(p)}$, we get $t_{0}>0$ such that $\varepsilon, \varepsilon^{\prime}, \ldots, \varepsilon^{(p)}$ are strictly monotone on $\left(t_{0}, \infty\right)$, tend to 0 at $\infty$, and $|\varepsilon(t)|<1, \ldots,\left|\varepsilon^{(p)}(t)\right|<1$, for all $|t|>t_{0}$. If we take a constant $M$ large enough, then $\varphi=\varepsilon^{p+1} / M$ has the desired properties.

For general $n$, let $\varepsilon_{1}(x)=\varepsilon(x) / N\left(1+\|x\|^{p}\right)$, where $N$ is a positive number. Let $\alpha(t)=\min \left\{\varepsilon_{1}(x):\|x\|^{2} \leq|t|\right\}$. Applying the case $n=1$, we have a positive definable $C^{p}$ function $g: \mathbb{R} \rightarrow \mathbb{R}$ such that $\left|g^{(k)}\right|<\alpha$ for all $k \in\{0, \ldots, p\}$. Now let $\varphi(x)=g\left(\|x\|^{2}\right)$. Then by the chain rule, when $N$ is large enough, we have

$$
\left|\partial^{\alpha} \varphi(x)\right|<N \varepsilon_{1}(x)\|x\|^{|\alpha|}<\varepsilon(x), \quad \forall x \in \mathbb{R}^{n}, \forall|\alpha|<p .
$$

Proof of Theorem 3. We divide the proof into two parts: density and openness.

Density. Let $f \in \mathcal{D}^{p}\left(\mathbb{R}^{n}\right)$, and $\varepsilon: \mathbb{R}^{n} \rightarrow \mathbb{R}$ be a positive continuous definable function. We will find a Morse function on $X$ in the $\varepsilon$-neighborhood of $f$.

Let $N=1+n 2^{p}$. By Lemma 4 , there exists a positive definable $C^{p}$ function $\varphi: \mathbb{R}^{n} \rightarrow \mathbb{R}$ such that

$$
\left|\partial^{\alpha} \varphi(x)\right|<\frac{\varepsilon(x)}{N(1+\|x\|)}, \quad \forall|\alpha| \leq p .
$$

Consider the family

$$
F: I^{n+1} \times \mathbb{R}^{n} \rightarrow \mathbb{R}, \quad F(t, x)=f_{t}(x)=f(x)+t_{0} \varphi(x)+\sum_{i=1}^{n} t_{i} x_{i} \varphi(x),
$$

where $I=(-1,1), t=\left(t_{0}, t_{1}, \ldots, t_{n}\right)$, and $x=\left(x_{1}, \ldots, x_{n}\right)$. To apply Theorem 2 , we check that

$$
\Phi(t, x)=\left(\sum_{j=1}^{n}\left(\frac{\partial f}{\partial x_{j}}(x)+t_{0} \frac{\partial \varphi}{\partial x_{j}}(x)+t_{j} \varphi(x)+\sum_{i=1}^{n} t_{i} x_{i} \frac{\partial \varphi}{\partial x_{j}}(x)\right) d x_{j}, x\right)
$$

is submersive. Indeed, since $\varphi(x) \neq 0$, the rank of the Jacobian $J \Phi(t, x)=$ 
$\left(\begin{array}{ccclcc}0 & 0 & 0 & \cdots & 0 & I_{n} \\ \frac{\partial \varphi}{\partial x_{1}}(x) & \varphi(x)+x_{1} \frac{\partial \varphi}{\partial x_{1}}(x) & x_{2} \frac{\partial \varphi}{\partial x_{1}}(x) & \cdots & x_{n} \frac{\partial \varphi}{\partial x_{1}}(x) & * \\ \frac{\partial \varphi}{\partial x_{2}}(x) & x_{1} \frac{\partial \varphi}{\partial x_{2}}(x) & \varphi(x)+x_{2} \frac{\partial \varphi}{\partial x_{2}}(x) & \cdots & x_{n} \frac{\partial \varphi}{\partial x_{2}}(x) & * \\ \vdots & \vdots & \vdots & & \vdots & \vdots \\ \frac{\partial \varphi}{\partial x_{n}}(x) & x_{1} \frac{\partial \varphi}{\partial x_{n}}(x) & x_{2} \frac{\partial \varphi}{\partial x_{n}}(x) & \cdots & \varphi(x)+x_{n} \frac{\partial \varphi}{\partial x_{n}}(x) & *\end{array}\right)$

is $n+\operatorname{rank}\left(\varphi^{\prime}(x), \varphi(x) e_{1}+x_{1} \varphi^{\prime}(x), \ldots, \varphi(x) e_{n}+x_{n} \varphi^{\prime}(x)\right)=2 n$ (where $e_{1}, \ldots, e_{n}$ is the standard basis of $\left.\mathbb{R}^{n}\right)$. So the set $\left\{t \in I^{n+1}: f_{t}\right.$ is Morse on $X\}$ is dense in $I^{n+1}$.

On the other hand, by Leibniz's rule, it is easy to see that for each $\alpha \in \mathbb{N}^{n}$ with $|\alpha| \leq p$, we have

$$
\begin{aligned}
\left|\partial^{\alpha}\left(f_{t}-f\right)(x)\right| & \leq\left|t_{0}\right|\left|\partial^{\alpha} \varphi(x)\right|+\sum_{i=1}^{n}\left|t_{i}\right|\left|\partial^{\alpha}\left(x_{i} \varphi\right)(x)\right| \\
& <\left(1+n 2^{p}\right) \varepsilon(x) / N=\varepsilon(x) .
\end{aligned}
$$

Therefore, there exists $t \in I^{n+1}$ such that $f_{t}$ is a Morse function on $X$ in the $\varepsilon$-neighborhood of $f$ in $\mathcal{D}^{p}\left(\mathbb{R}^{n}\right)$.

To get a Morse function with distinct critical values, we construct it as follows. Suppose $f$ is a Morse function on $X$. For each $S \in \mathcal{S}$, the set of critical points of $\left.f\right|_{S}$ is finite, because it is definable and discrete. So $f$ has only finitely many critical points on $X$. Let $x_{1}, \ldots, x_{q}$ be the critical points for $\left.f\right|_{S}$, for all $S$ in $\mathcal{S}$. Let $r>0$ be small enough so that the balls $B\left(x_{i}, r\right), i=1, \ldots, q$, are disjoint. For $i=1, \ldots, q$, choose a definable $C^{p}$ function $\lambda_{i}: \mathbb{R}^{n} \rightarrow[0,1]$ such that $\lambda_{i}=0$ on $\mathbb{R}^{n} \backslash B\left(x_{i}, r\right)$, and $\lambda_{i}(x)=1$ on $B\left(x_{i}, r / 2\right)$. Consider the approximations of $f$ of the form

$$
g=f+c_{1} \lambda_{1}+\cdots+c_{q} \lambda_{q} .
$$

Then in any neighborhood $U$ of $f$, we can choose $c_{1}, \ldots, c_{q}$ so that $g \in U, g$ is a Morse function on $X$ with the set of critical points being $\left\{x_{1}, \ldots, x_{q}\right\}$, and $g\left(x_{i}\right) \neq g\left(x_{j}\right)$ when $i \neq j$. This completes the proof of the density part.

Openness. Let $f \in \mathcal{D}^{p}\left(\mathbb{R}^{n}\right)$ be a Morse function on $X$ with distinct critical values. We will find a neighborhood of $f$ that contains only Morse functions on $X$ with distinct critical values.

By Lemma 2, there is a positive continuous definable function $m_{f}$ : $\mathbb{R}^{n} \rightarrow \mathbb{R}$ such that for each $S \in \mathcal{S}$,

$$
m_{f}(x) \leq\left\|\left.d f(x)\right|_{T_{x} S}\right\|+\left|\operatorname{det} d^{2} f(x)\right|_{T_{x} S} \mid \frac{d(x, \partial S)}{1+d(x, \partial S)}, \quad \forall x \in S .
$$

Define $\varepsilon_{X}=\frac{1}{3} m_{f}$. 
By Lemmas 1 and 3, there exists a positive continuous semialgebraic function $\delta: \mathbb{R}_{+} \times L_{2}\left(\mathbb{R}^{n}, \mathbb{R}\right) \rightarrow \mathbb{R}$ satisfying the following condition for all $k \in\{0, \ldots, n\}$ and all $T \in G_{k}\left(\mathbb{R}^{n}\right)$ :

$$
B^{\prime} \in L_{2}\left(\mathbb{R}^{n}, \mathbb{R}\right),\left\|B-B^{\prime}\right\|<\delta(\varepsilon, B) \Rightarrow|\operatorname{det} B|_{T}-\left.\operatorname{det} B^{\prime}\right|_{T} \mid<\varepsilon .
$$

Let $\varepsilon=\min \left\{\varepsilon_{X}, \delta\left(\varepsilon_{X}, d^{2} f\right)\right\}$. Then $\varepsilon$ is a positive continuous definable function on $\mathbb{R}^{n}$. By the construction of $\varepsilon$, if $g \in \mathcal{D}^{p}\left(\mathbb{R}^{n}\right),\|d g-d f\|<\varepsilon$ and $\left\|d^{2} g-d^{2} f\right\|<\varepsilon$, then for each $x \in S \in \mathcal{S}$,

$$
\begin{aligned}
m_{g, S}(x) & =\left\|\left.d g(x)\right|_{T_{x} S}\right\|+\left|\operatorname{det} d^{2} g(x)\right|_{T_{x} S} \mid \frac{d(x, \partial S)}{1+d(x, \partial S)} \\
& >m_{f, S}(x)-2 \varepsilon_{X}(x) \geq \varepsilon(x) .
\end{aligned}
$$

So, by Lemma 2, $g$ is a Morse function on $X$.

Moreover, since $f$ has only a finite number of critical points and takes distinct values at them, we can reduce $\varepsilon$ so that if $g \in \mathcal{D}^{p}\left(\mathbb{R}^{n}\right),|g-f|<\varepsilon$, $\|d g-d f\|<\varepsilon$, and $\left\|d^{2} g-d^{2} f\right\|<\varepsilon$, then $g$ is Morse on $X$, the set of critical points of $\left.g\right|_{X}$ is close to that of $f$, and $g$ still has distinct critical values. We have constructed a neighborhood of $f$ in $\mathcal{D}^{p}\left(\mathbb{R}^{n}\right)$ containing only Morse functions on $X$ with distinct critical values.

To apply Morse theory to definable sets, one needs the following corollary:

Corollary 3. There exists a definable $C^{p}$ Morse function on $X$ which is proper and has distinct critical values.

Proof. By Corollary 1, there exists a definable $C^{p}$ Morse function $f$ on $X$ which is proper. An approximation of $f$ which has distinct critical values is constructed in the proof of Theorem 3.

Using the same arguments as in $[\mathrm{P}$, Th. 2] one obtains:

Corollary 4. If $f \in \mathcal{D}^{p}\left(\mathbb{R}^{n}\right)(p \geq 3)$ is a Morse function on $X$ which is proper and has distinct critical values, then $f$ is stable in the sense that there exists an open neighborhood $U$ of $f$ in $\mathcal{D}^{p}\left(\mathbb{R}^{n}\right)$ such that for each $g \in U$, one can find homeomorphisms $h: X \rightarrow X$ and $\lambda: \mathbb{R} \rightarrow \mathbb{R}$ such that $g \circ h=\lambda \circ f$.

REMARK 4. For the density of Morse functions to be true, $X$ is not required to be closed. However, for the openness to be true, $X$ must be closed and the stratification must satisfy Whitney's condition $A$ (see an example in $[\mathrm{P}]$, see also $[\mathrm{T}])$.

REMARK 5. Our proof of Theorem 3 can be generalized to obtain Thom's transversality theorem in the o-minimal context. We also refer the reader to $[\mathrm{S}]$ for a version of transversality theorem. 


\section{References}

[Be] R. Benedetti, Density of Morse functions on complex spaces, Math. Ann. 229 (1977), 135-139.

[Br] J. W. Bruce, Generic functions on semialgebraic sets, Quart. J. Math. Oxford (2) 37 (1986), 137-165.

[C] M. Coste, An Introduction to o-Minimal Geometry, Doctorato di Ricerca in Matematica, Dip. Mat. Pisa, Instituti Editoriali e Poligrafici Internazionali, 2000.

[D] L. van den Dries, Tame Topology and o-Minimal Structures, London Math. Soc. Lecture Note Ser. 248, Cambridge Univ. Press, 1997.

[DM] L. van den Dries and C. Miller, Geometric categories and o-minimal structures, Duke Math. J. 84 (1996), 497-540.

[E] J. Escribano, Approximation theorems in o-minimal structures, Illinois J. Math. 46 (2002), 111-128.

[GM] M. Goresky and R. MacPherson, Stratified Morse Theory, Springer, 1988.

[La] F. Lazzeri, Morse theory on singular spaces, Astérisque 7-8 (1973), 263-268.

[Lo] T. L. Loi, Verdier stratifications and Thom stratifications in o-minimal structures, Illinois J. Math. 42 (1998), 347-356.

[Mi] J. Milnor, Morse Theory, Princeton Univ. Press, 1963.

[Mo] M. Morse, The critical points of a function of $n$ variables, Trans. Amer. Math. Soc. 33 (1931), 72-91.

[O] P. Orro, Conditions de regularité, espaces tangents et fonctions de Morse sur les espaces stratifiés, thesis, Univ. of Paris, Orsay, 1984.

[P] R. Pignoni, Density and stability of Morse functions on a stratified space, Ann. Scuola Norm. Sup. Pisa (4) 4 (1979), 529-608.

[S] M. Shiota, Geometry of Subanalytic and Semialgebraic Sets, Progr. Math. 150, Birkhäuser, Boston, 1997.

[T] D. J. A. Trotman, Stability of transversality to a stratification implies Whitney A-regularity, Invent. Math. 50 (1979), 273-277.

[Wh] H. Whitney, Tangents to an analytic variety, Ann. of Math. 81 (1965), 496-549.

[Wi] A. J. Wilkie, A theorem of the complement and some new o-minimal structures, Selecta Math. (N.S.) 5 (1999), 397-421.

Department of Mathematics

University of Dalat

Dalat, Vietnam

E-mail: taleloi@hotmail.com

Received 12.7.2005

and in final form 27.7.2006 